\title{
Nice Pairs of Pentagons in Chamfered Fullerenes
}

\author{
Meysam Taheri-Dehkordi, Gholam Hossein Fath-Tabar \\ Department of Pure Mathematics, Faculty of Mathematical Sciences, \\ University of Kashan, Kashan 87317-53153, Iran
}

(Received September 14, 2021)

\begin{abstract}
Fullerenes graphs are 3-connected, 3-regular planar graphs with faces including only pentagons and hexagons. If $G$ be a graph with a perfect matching, a subgraph $H$ of $G$ is a nice subgraph if $G-V(H)$ has a perfect matching. In this paper, we show that in every fullerene graph arising from smaller fullerenes via chamfer transformation, each pair of pentagons is a nice subgraph.
\end{abstract}

\section{Introduction}

The first fullerene molecule was discovered in 1985 [9], and since then, the fullerenes have been objects of interest to scientists all over the world. Many mathematical properties of fullerene molecules can be studied using mathematical methods. It follows from the Euler formula that there must be exactly 12 pentagons in every fullerene graph, while there is no condition on the number of hexagons. The number of vertices in fullerenes is necessarily even, and the existence of such graphs on $n$ vertices was established for all even $n \geq 20$ except $n=$ 22 in a classical paper by Grünbaum and Motzkin [7]. A classical survey of early results on fullerene graphs is [6], and for a more recent comprehensive review, we refer the reader to [11]. We denote respectively by $E(G)$ and $V(G)$ the set of edges and vertices of a graph $G$. 
One of the most important structures in the chemical study of fullerenes is the Kekulé structure, which is defined as perfect matching in graph theory. In $[4,5]$ the reader can read more about perfect matching in fullerene graphs.

All nice subgraphs must have an even number of vertices. In section 3 we discuss the case where the nice subgraph includes two pentagons.

\section{Definitions and auxiliary Results}

Many fullerene graphs arise from smaller fullerene graphs by applying some transformation. The number of vertices in the resulting graph is usually an integer multiple of the number of vertices of starting graph. The best known such transformation is the leapfrog transformation that can be thought of as truncation of the dual. [6]

One of the less known but still very useful is known as chamfer, resulting in fullerene graphs with four times the number of vertices. We refer the reader to [8] to read more about these transformations and other transformations. We will continue this section with how to work chamfer transformation. Consider the Fullerene graph $G$, where in each face of $G$ we draw a similar face. These faces are shown in Fig 1 . Then connect each vertex of the original fullerene with three vertices of newly inscribed polygons, one vertex in each of the faces with which they are incident (bold full lines in Fig. 1).



Figure 1. Chamfer transformation. 
Finally, erase the edges of the original fullerene. The resulting graph has only pentagonal and hexagonal faces. The number of pentagonal faces is exactly twelve. The number of vertices is four times the number of vertices in the original fullerene. As the resulting graph is clearly planar, cubic, and 3 connected, it is a fullerene graph. The resulting fullerene is called chamfered fullerene.

Theorem 1.[1] Let $G$ be a leapfrog fullerene. Then, any two pentagons in $G$ form a nice pair.

In [1] it is proven that all pairs of pentagons in Leapfrog Fullerenes are nice. We extened this result for chamfered fullerenes (Fullerenes obtained the chamfering transformation from smaller fullerenes) after the fashion of [1].

\section{Main Results}

In [2] is shown that in all isolated pentagon (IP) fullerenes on 100 vertices at most, all pairs of pentagons are nice. From this, we can conclude the following statement

Proposition 2. All pairs of pentagons in Chamfered fullerenes $\boldsymbol{C}_{\mathbf{8 0}}$ and $\boldsymbol{C}_{\mathbf{9 6}}$ are nice.

Theorem 3. Let $G$ be a chamfered fullerene. Then, all pairs of pentagons in $G$ are nice.

Proof. We know that all fullerene graphs have perfect matchings, it follows from a classical result of Petersen that every connected cubic graph with no more than two cut-edges has a perfect matching [10].

First, we present a method for a perfect matching in chamfered fullerenes.

According to the method of producing chamfered fullerenes, if there are $h$ hexagons between two pentagons in the original graph, after performing the chamfer transformation, the number of $\lambda$ hexagons will be between those two pentagons in the chamber fullerene. And we have

$$
\lambda=2 h+1
$$

Therefore, the structure of faces in chamfered fullerenes is the same as in Fig 2 

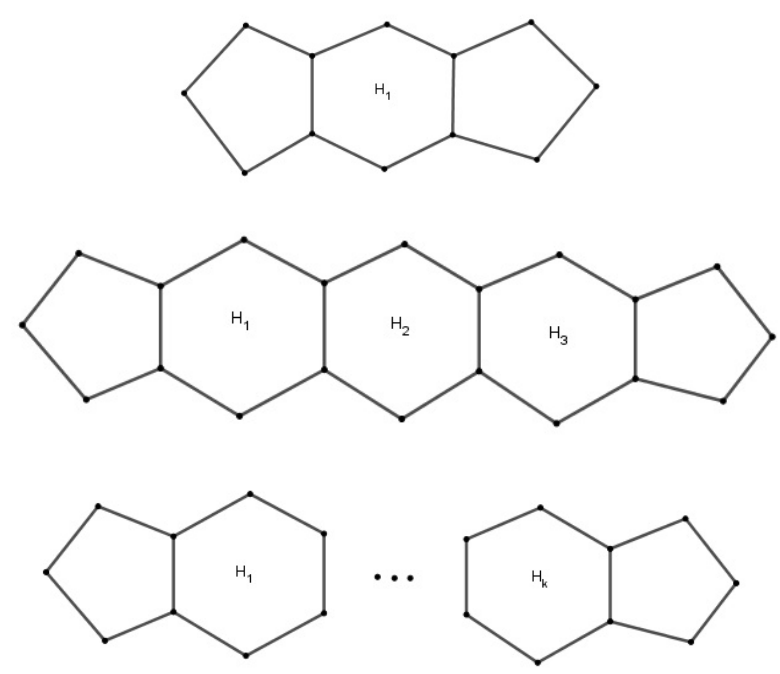

Figure 2. Placement of faces in chamfered fullerenes.

The number of hexagons between the pentagons is an odd number $\lambda$. We now consider two modes:

$\lambda=1$. In this case, According to [3], we know that all chamfered fullerenes have perfect star packaging. we consider perfect star packaging $M$ for $G$ as follows: Consider vertices $v_{1}$ and $v_{2}$ on the pentagons $P_{1}$ and $P_{2}$ as the central vertices of the star, On the other hand, Each pentagon can contain at most one center of a star. Therefore perfect star packing $M$ can be considered as Figure 3. 




Figure 3. Perfect star packaging $M$ of $G$.

Using this packing, we define a matching $M^{\prime}$ for $G$ as shown by the dashed lines in Figure 4: The edges of stars $S_{1}$ and $S_{2}$ are not, in edges of $M^{\prime}$ and one edge of all the other stars should be considered as a matching edge $M^{\prime}$.

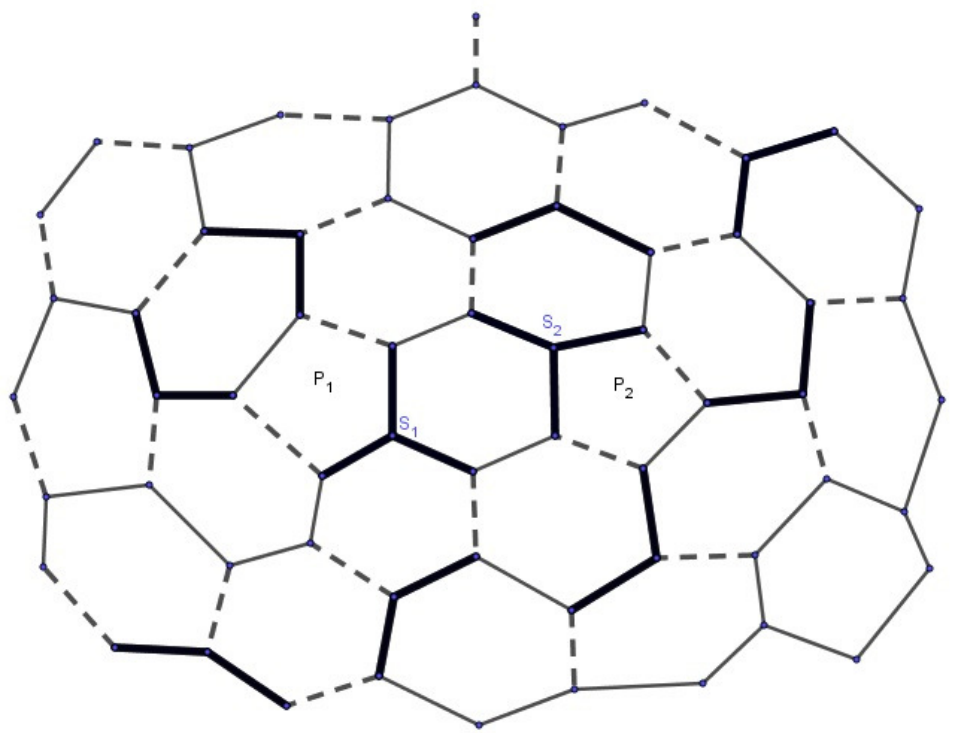

Figure 4. Dashed edges are matching edges. 
Now if we remove the vertices of pentagons $P_{1}$ and $P_{2}$, we will have a perfect matching for $G-V\left(P_{1}\right)-V\left(P_{2}\right)$.

$\lambda \geq 3$. In this case, a perfect matching can be considered as follows

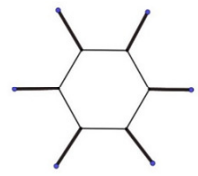

Figure 5. Perfect matching edges in pentagons (Tick lines).

In hexagons, the desired matching occurs in the following three ways that are shown in fig 6


Figure 6. Perfect matching edges in hexagons (Tick lines).

For example, for $\lambda=5$ mode, we have the following figure

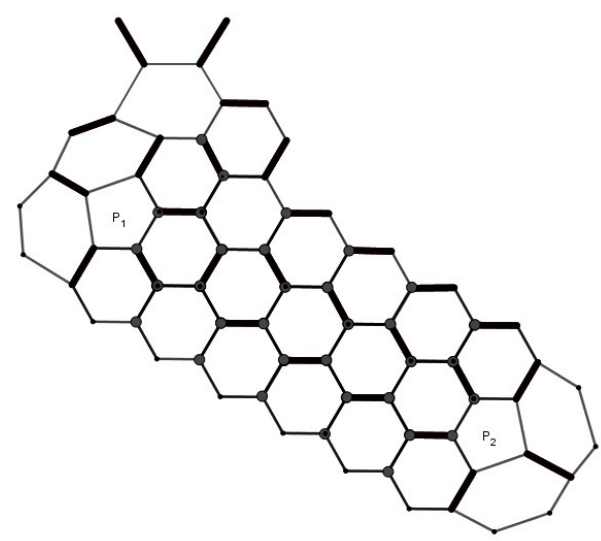

Figure 7. Perfect matching edges for $\lambda=5$. 
Let $G$ be a chamfered fullerene and $P_{1}, P_{2}$ be two of its pentagon faces and $M$ be the perfect matching as defined above that shown in fig 7, and the edges of the original fullerene are shown by thin dashed line.



Figure 8.

Consider the shortest path connecting pentagons in the original fullerene; the path is indicated by thick dashed lines in Figure 8 . The length of this path in the original Fullerene is equal to $2 h$. This path in the original fullerene corresponds to four numbers of adjacent hexagons $H_{1}, H_{2}$, $H_{3}, H_{4}$ in the chamfered fullerene. We construct an $M$-alternating path $P$ of odd length connecting a vertex $u$ of $P_{1}$ to a vertex $v$ in $P_{2}$

$$
P: u-a-b-c-d-e-f-v
$$

By flipping the edges on path $P$ and the four hexagons $A_{1}, A_{2}, A_{3}, A_{4}$, we obtain a matching, called $M^{\prime}$, in $G$ that matches no vertices from $G-V\left(P_{1}\right)-V\left(P_{2}\right)$ to vertices on $P_{1}$ or $P_{2}$. The situation is shown in Figure 9.

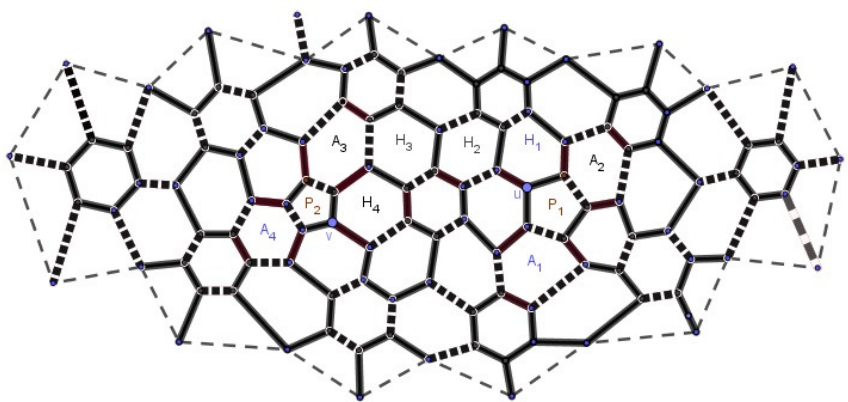

Figure 9. Edges of Matching $M^{\prime}$ are shown in thick dashed lines. 
Acknowledgment: The research reported in this paper was partially supported by University of Kashan under Grant number 985985/5.

\section{References}

[1] T. Došlić, All pairs of pentagons in leapfrog fullerenes are nice, Mathematics 8 (2020) \#2135.

[2] T. Došlić, Nice pairs of odd cycles in fullerene graphs, J. Math. Chem. 58 (2020) 2204 2222.

[3] T. Došlić, M. T. Dehkordi, G. H. Fath-Tabar, Packing stars in fullerenes, J. Math. Chem. 58 (2020) 2223-2244.

[4] T. Došlić, On some structural properties of fullerene graphs, J. Math. Chem. 31 (2002) $187-195$.

[5] T. Došlić, On lower bounds of number of perfect matchings in fullerene graphs, J. Math. Chem. 24 (1998) 359-364.

[6] P. W. Fowler, D. E. Manolopoulos, An Atlas of Fullerenes, Clarendon Press, Oxford, 1995.

[7] B. Grünbaum, T. S. Motzkin, The number of hexagons and the simplicity of geodesics on certain polyhedra, Can. J. Math. 15 (1963) 744-751.

[8] R. B. King, M. V. Diudea, The chirality of icosahedral fullerenes: A comparison of the tripling, (leapfrog), quadrupling (chamfering) and septupling (capra) transformations, $J$. Math. Chem. 39 (2006) 597-604.

[9] H. W. Kroto, J. R. Heath, S. C. O’Brien, R. F. Curl, R. E. Smalley, C60 Buckminsterfullerene, Nature 318 (1985) 162-163.

[10] J. Petersen, Die Theorie der regulären graphs, Acta Math. 15 (1891) 193-220.

[11] P. Schwerdtfeger, L. N. Wirz, J. Avery, The topology of fullerenes, WIREs 5 (2015) 96145 . 